Brief paper

\title{
Least-restrictive robust periodic model predictive control applied to room temperature regulation
}

\author{
Ravi Gondhalekar ${ }^{\mathrm{a}, 1}$, Frauke Oldewurtel $^{\mathrm{b}}$, Colin N. Jones ${ }^{\mathrm{c}}$ \\ a Department of Chemical Engineering, University of California Santa Barbara (UCSB), USA \\ ${ }^{\mathrm{b}}$ Power Systems Laboratory, Swiss Federal Institute of Technology Zurich (ETHZ), Switzerland \\ ${ }^{\mathrm{c}}$ Automatic Control Laboratory, Department of Mechanical Engineering, École Polytechnique Fédérale de Lausanne (EPFL), Switzerland
}

\section{A R T I C L E I N F O}

\section{Article history:}

Received 28 November 2011

Received in revised form

19 December 2012

Accepted 2 May 2013

Available online 14 June 2013

\section{Keywords:}

Model predictive control

Constrained control

Robust control

Periodic control

Building climate control

\begin{abstract}
A B S T R A C T
State-feedback model predictive control (MPC) of constrained discrete-time periodic affine systems is considered. The periodic systems' states and inputs are subject to periodically time-dependent, hard, polyhedral constraints. Disturbances are additive, bounded and subject to periodically time-dependent bounds. The objective is to design MPC laws that robustly enforce constraint satisfaction in a manner that is least-restrictive, i.e., have the largest possible domain. The proposed design method is demonstrated on a building climate control example. The proposed method is directly applicable to time-invariant MPC.
\end{abstract}

(c) 2013 Elsevier Ltd. All rights reserved.

\section{Introduction}

In this paper an approach is proposed for performing robust constrained MPC in a manner that is least-restrictive, i.e., the controller domain is as large as possible. The theory of constrained MPC tends to focus on enforcing some notion of stability or optimality (Mayne, Rawlings, Rao, \& Scokaert, 2000), where this may be achieved at the expense of small controller domains. When the set-point is far within the constraints there are no consequences of not being least-restrictive. However, the consideration of leastrestrictive robust control is crucial when the control performance is heavily influenced by the ability to operate the plant close to the constraints - MPC's original motivation - e.g., when the reference trajectory itself is not admissible (see Section 5). In the proposed approach maximum robust (periodic) controlled invariant sets (see Definition 3 and Blanchini \& Ukovich, 1993) are employed in the

\footnotetext{
This work was supported in part by the Program of Promotion of Environmental Improvement to Enhance Young Researchers' Independence under the Special Coordination Funds for Promoting Science and Technology, Japan Ministry of Education, Culture, Sports, Science and Technology. The material in this paper was partially presented at the 49th IEEE Conference on Decision and Control (CDC), December 15-17, 2010, Atlanta, Georgia, USA. This paper was recommended for publication in revised form by Associate Editor Martin Guay under the direction of Editor Frank Allgöwer.

E-mail addresses: gondhalekar@engineering.ucsb.edu (R. Gondhalekar), oldewurtel@eeh.ee.ethz.ch (F. Oldewurtel), colin.jones@epfl.ch (C.N. Jones).

1 Tel.: +1 805893 3120; fax: +1 8058934731 .
}

design of least-restrictive robustly strongly feasible (periodic) MPC problems, i.e., constrained MPC problems that indefinitely enforce recursive feasibility for the receding-horizon application of any feasible solution (see Section 4.2 and Kerrigan, 2000). The proposed control strategy performs so-called zone-MPC (Ferramosca, Limon, González, Odloak, \& Camacho, 2010; Grosman, Dassau, Zisser, Jovanovič, \& Doyle, 2010; Maciejowski, 2002) that focuses on containment of the state in a specified region; a priori guarantees of stabilization or optimality with respect to a set-point or reference trajectory are not provided by the proposed method. However, the MPC problem's cost function and prediction horizon length, both crucial handles for tuning the performance and complexity of an MPC controller, can be adjusted at will in order to achieve good cost performance and stability with respect to a set-point or state-reference trajectory, as can be verified a posteriori. Due to the robust strong feasibility of the MPC problem, this tuning can be performed freely without jeopardizing robust constraint satisfaction.

Periodic systems are an important extension of time-invariant systems and provide a natural framework for modeling various real-world phenomena and control problems (Bittanti \& Colaneri, 2009; Gondhalekar \& Jones, 2011; Varga, 2007). This paper treats periodic affine systems; useful generalizations of periodic linear systems. Conveniently, many tools for rigorously performing constrained MPC of periodic affine systems, e.g., invariant set methods (Blanchini, 1999; Blanchini \& Ukovich, 1993) and convex optimization (Boyd \& Vandenberghe, 2004), can be derived from 
the well-established equivalent tools for linear systems. The equivalent exact tools are not available for general nonlinear systems, and thus in this paper the conservativeness and complication of general nonlinear MPC methods are avoided. However, the concepts behind the proposed methods are applicable to general systems. The class of periodic affine systems subsumes linear periodic and linear time-invariant (LTI) systems, so the proposed methods are directly applicable to those, more commonly considered, cases. It seems that periodic control formulations are on occasion intentionally avoided, because they seem difficult or not possible. This is especially so for the unusual case considered in this paper (see also Gondhalekar \& Jones, 2011), where the systems' state and input dimensions are time-dependent. A strong motivation for this paper was to demonstrate that a robust periodic constrained MPC formulation is both straightforward and also very useful to consider.

The contribution, and novelty, of this paper is thus twofold. The primary contribution is to propose a method to perform least-restrictive robust constrained MPC. The second is to present the proposed least-restrictive robust constrained MPC approach in a manner applicable to certain classes of periodic systems. The proposed strategy is a combination, and extension to robust control, of the least-restrictive nominal strongly feasible moveblocking MPC problem design of Gondhalekar and Imura (2010), and the method proposed in Gondhalekar and Jones (2011) for nominal linear periodic MPC.

The proposed MPC strategy is demonstrated in Section 5 on a building climate control problem, where the room temperature is to be controlled to satisfy comfort constraints. The first important property of the example is that the room temperature is constrained to comfort zones, not (always) a specific set-point. Secondly, the input reference is zero (ideally no heating/cooling would need to be performed), but may not be indefinitely admissible. The reason for applying a non-zero control input is only to enforce the constraints. Thus it is desirable to allow, at each moment in time, the room to be as cold (warm) as possible, and only heat (cool) as necessary to avoid constraint violations. The notion of least-restrictive control is of relevance here. Third, the consideration of periodic control for building climate control is useful despite the heat-transfer dynamics of a building being time-invariant. This is because the environmental factors affecting the system, and also the buildings' occupancy pattern, are periodically time-dependent.

Notation: The set of reals is denoted by $\mathbb{R}$, the set of non-negative integers by $\mathbb{N}\left(\mathbb{N}_{+}:=\mathbb{N} \backslash\{0\}\right)$, the set of non-negative integers $\{j, \ldots, k\}$ by $\mathbb{N}_{j}^{k}$, the $n \times n$ identity matrix by $I_{n}$, the zero matrix with appropriate dimension by 0 , and a sequence of elements $x_{i} \in$ $\mathbb{X} \forall i \in \mathbb{N}_{j}^{k}$ by $\left\{x_{i} \in \mathbb{X}\right\}_{i=j}^{k}$. For sets $\mathbb{A}, \mathbb{B} \subseteq \mathbb{R}^{n}$ let $\mathbb{A} \ominus \mathbb{B}:=\{x \in$ $\left.\mathbb{R}^{n} \mid x+b \in \mathbb{A} \forall b \in \mathbb{B}\right\}$. Let $\psi_{(i, k)}$ denote the future value of $\psi$ at step $i+k$, as predicted from step $i$. Let $\psi_{i}:=\psi_{(i, 0)}$.

\section{Problem setting}

We consider the discrete-time periodic affine system (1) with time step $i \in \mathbb{N}$, period length $p \in \mathbb{N}_{+}$, and intra-period stepindex function $\sigma(\cdot)$. As the time step $i$ progresses from 0 toward $\infty$ the subscript $\sigma(i)$ on the affine dynamics matrices $A, B, C$ and $c$ (later also other variables) repeatedly cycles through the sequence $0,1, \ldots, p-1$, thereby inducing the periodic time-dependence in the dynamics. The system state is denoted by $x_{i} \in \mathbb{R}^{n_{\sigma(i)}}$, the control input by $u_{i} \in \mathbb{R}^{m_{\sigma(i)}}$, and the additive disturbance by $w_{i} \in \mathbb{R}^{v_{\sigma(i)}}$. The dimensions of state, input and disturbance vectors are also periodically time-dependent and satisfy $n_{j}, m_{j}, v_{j} \in \mathbb{N}_{+} \forall j \in \mathbb{N}_{0}^{p-1}$. Such periodic systems are unusual but make convenient models of systems with asynchronous control inputs (Gondhalekar \& Jones, 2011). Furthermore, minimal periodic system realizations generally require time-dependent dimensions (Varga, 2004). If $m_{\sigma(i)}=0$ then system (1) is autonomous at step $i$, and it is useful to accommodate such cases (Gondhalekar \& Jones, 2011). However, to avoid notational abuses, e.g., $B_{\sigma(i)} \in \mathbb{R}^{n_{\sigma(i+1)} \times 0}$, the exposition here is restricted to systems with strictly positive input dimensions. The application of the proposed method to systems with piecewise autonomous behavior is immediate and not discussed further:

$x_{i+1}=A_{\sigma(i)} x_{i}+B_{\sigma(i)} u_{i}+C_{\sigma(i)} w_{i}+c_{\sigma(i)}$

$\sigma(i):=i$ modulo $p, \quad \sigma: \mathbb{N} \rightarrow \mathbb{N}_{0}^{p-1}$.

The states and inputs are required to satisfy the periodically timedependent, convex, polyhedral constraints

$E_{\sigma(i)} x_{i}+G_{\sigma(i)} u_{i} \leq b_{\sigma(i)}$.

Assumption 1. Disturbances satisfy $w_{i} \in \mathbb{W}_{\sigma(i)}$, where $\mathbb{W}_{j} \subset$ $\mathbb{R}^{v_{j}}, 0 \in \mathbb{W}_{j} \forall j \in \mathbb{N}_{0}^{p-1}$ are non-empty polytopes.

The assumption that $0 \in \mathbb{W}_{j}$ is made without loss of generality. If $0 \notin \mathbb{W}_{j}$ then one can select any $\bar{w} \in \mathbb{W}_{j}$ and employ affine term $\bar{c}_{j}:=c_{j}+\bar{w}$ and disturbance set $\overline{\mathbb{W}}_{j}:=\left(\mathbb{W}_{j} \ominus\{\bar{w}\}\right) \ni 0$.

A periodic state-feedback control law is denoted by the set of functions $\left\{\kappa_{0}(\cdot), \ldots, \kappa_{p-1}(\cdot)\right\}, \kappa_{j}: \mathbb{R}^{n_{j}} \rightarrow \mathbb{R}^{m_{j}} \forall j \in \mathbb{N}_{0}^{p-1}$, such that $u_{i}=\kappa_{\sigma(i)}\left(x_{i}\right)$.

\section{Robust periodic controlled set invariance}

In this section (maximum) robust periodic controlled invariant sets of system (1) subject to (2) are defined for later use. The definitions are a minor generalization of the (maximum) robust periodic controlled set invariance notion proposed in Blanchini and Ukovich (1993), Gondhalekar and Jones (2011). The numerical characterization of these sets can be performed using the method described in Blanchini and Ukovich (1993), but for brevity the algorithm is not described further, as it is not a contribution of this paper.

Definition 2. A set $\left\{\mathbb{C}_{0}, \ldots, \mathbb{C}_{p-1}\right\}$ of sets $\mathbb{C}_{j} \subseteq \mathbb{R}^{n_{j}} \forall j \in \mathbb{N}_{0}^{p-1}$ is a robust periodic controlled invariant set of (1) subject to (2) if and only if: $\exists \kappa_{j}: \mathbb{R}^{n_{j}} \rightarrow \mathbb{R}^{m_{j}}$ s.t. $\left[E_{j} x+G_{j} \kappa_{j}(x) \leq b_{j}\right] \wedge\left[A_{j} x+B_{j} \kappa_{j}(x)+\right.$ $\left.C_{j} w+c_{j} \in \mathbb{C}_{\sigma(j+1)}\right] \forall w \in \mathbb{W}_{j} \forall x \in \mathbb{C}_{j} \forall j \in \mathbb{N}_{0}^{p-1}$.

Definition 3. The maximum robust periodic controlled invariant set is the set $\left\{\mathbb{C}_{0}^{*}, \ldots, \mathbb{C}_{p-1}^{*}\right\}$ of sets $\mathbb{C}_{j}^{*}$ :

$$
\begin{aligned}
\mathbb{C}_{j}^{*}:= & \left\{x_{j} \in \mathbb{R}^{n_{j}} \mid \exists \kappa_{k}: \mathbb{R}^{n_{k}} \rightarrow \mathbb{R}^{m_{k}} \forall k \in \mathbb{N}_{0}^{p-1}\right. \text { s.t. } \\
& x_{i+1}=A_{\sigma(i)} x_{i}+B_{\sigma(i)} \kappa_{\sigma(i)}\left(x_{i}\right)+C_{\sigma(i)} w_{i}+c_{\sigma(i)} \\
& \wedge E_{\sigma(i)} x_{i}+G_{\sigma(i)} \kappa_{\sigma(i)}\left(x_{i}\right) \leq b_{\sigma(i)} \\
& \left.\forall w_{i} \in \mathbb{W}_{\sigma(i)} \forall i \in \mathbb{N}_{j}^{\infty}\right\} \quad \forall j \in \mathbb{N}_{0}^{p-1} .
\end{aligned}
$$

The above expression states that the sets $\mathbb{C}_{j}^{*}$ constituting the maximum robust periodic controlled invariant set are the sets of all initial states $x_{j}$ such that there exists a periodic control law $\left\{\kappa_{0}(\cdot), \ldots, \kappa_{p-1}(\cdot)\right\}$, such that applying it achieves constraint satisfaction indefinitely.

Assumption 4. $\mathbb{C}_{j}^{*} \neq \varnothing \forall j \in \mathbb{N}_{0}^{p-1}$, i.e., the maximum robust periodic controlled invariant set exists.

There exists no simple test (to the authors' knowledge) for verifying Assumption 4. This is a general limitation with robust invariant sets and is not due to the unusual periodic framework considered here. The authors' approach is to run the algorithm of Blanchini and Ukovich (1993) and check the resulting sets. If the condition of Assumption 4 is false then any attempt at a robust control formulation is futile. 
Let $\overline{\mathbb{C}}_{\sigma(j+1)}^{*}:=\mathbb{C}_{\sigma(j+1)}^{*} \ominus C_{j} \mathbb{W}_{j} \forall j \in \mathbb{N}_{0}^{p-1}$. For all $j \in \mathbb{N}_{0}^{p-1}$ it holds that $\left[x+C_{j} w \in \mathbb{C}_{\sigma(j+1)}^{*} \forall w \in \mathbb{W}_{j}\right] \Longleftrightarrow\left[x \in \overline{\mathbb{C}}_{\sigma(j+1)}^{*}\right]$. Note that $\overline{\mathbb{C}}_{j}^{*} \subseteq \mathbb{C}_{j}^{*} \forall j \in \mathbb{N}_{0}^{p-1}$ because $0 \in \mathbb{W}_{j} \forall j \in \mathbb{N}_{0}^{p-1}$ by Assumption 1 . Note also that $\overline{\mathbb{C}}_{j}^{*} \neq \emptyset \forall j \in \mathbb{N}_{0}^{p-1}$ due to Assumption 4. Further let the sets $\mathbb{X}_{j} \subseteq \mathbb{R}^{n_{j}} \forall j \in \mathbb{N}_{0}^{p-1}$ satisfy $\mathbb{X}_{j} \supseteq \mathbb{C}_{j}^{*} \forall j \in \mathbb{N}_{0}^{p-1}$. The $\overline{\mathbb{C}}_{j}^{*}$ and $\mathbb{X}_{j}$ sets are employed later in Problem 6.

\section{Periodic MPC}

In this section the MPC problem is formulated and discussed. The main design objective is the design of least-restrictive robust periodic control laws (see Definition 5). The design of leastrestrictive robust periodic control laws was previously considered in Blanchini and Ukovich (1993), albeit in a slightly different problem setting, and not in an MPC context.

Definition 5. A periodic state-feedback control law $\left\{\kappa_{0}(\cdot), \ldots\right.$, $\left.\kappa_{p-1}(\cdot)\right\}$ is termed a least-restrictive robust periodic control law if and only if it renders the maximum robust periodic controlled invariant set robustly controlled invariant by an admissible control input, i.e.: $\left[A_{j} x+B_{j} \kappa_{j}(x)+C_{j} w+c_{j} \in \mathbb{C}_{\sigma(j+1)}^{*}\right] \wedge\left[E_{j} x+G_{j} \kappa_{j}(x) \leq b_{j}\right]$ $\forall w \in \mathbb{W}_{j} \forall x \in \mathbb{C}_{j}^{*} \forall j \in \mathbb{N}_{0}^{p-1}$.

\subsection{Periodic MPC problem}

For a prediction horizon length $N \in \mathbb{N}_{+}$, MPC achieves closed-loop control action of system (1) subject to constraints (2) by applying at each state $x_{i}=x_{(i, 0)}$ the first control input $u_{(i, 0)}$ of the predicted optimal control input trajectory $\bar{u}_{i}^{*}\left(x_{i}\right):=$ $\left[u_{(i, 0)}^{\top}, \ldots, u_{(i, N-1)}^{\top}\right]^{\top} \in \mathbb{R}^{\bar{m}_{j}}, \bar{m}_{j}:=\sum_{i=j}^{j+N-1} m_{\sigma(i)} \forall j \in \mathbb{N}_{0}^{p-1}$. Note that $N$ can be chosen independently of period length $p$. The optimization is performed with respect to the periodic cost function $J_{j}: \mathbb{R}^{n_{j}} \times \mathbb{R}^{\bar{m}_{j}} \rightarrow \mathbb{R} \forall j \in \mathbb{N}_{0}^{p-1}\left(\operatorname{recall} x_{(i, 0)}:=x_{i}\right)$ :

$$
\begin{aligned}
J_{\sigma(i)}\left(x_{i}, \bar{u}_{i}\right):= & \sum_{k=0}^{N-1} R_{\sigma(i+k)} u_{(i, k)}+\sum_{k=0}^{N}\left(x_{(i, k)}-r_{\sigma(i+k)}\right)^{\top} Q_{\sigma(i+k)} \\
& \times\left(x_{(i, k)}-r_{\sigma(i+k)}\right) .
\end{aligned}
$$

The input costs are linear with periodic cost matrices $R_{j} \in \mathbb{R}^{1 \times m_{j}}$ $\forall j \in \mathbb{N}_{0}^{p-1}$, the state-error cost is quadratic with periodic cost matrices $Q_{j} \in \mathbb{R}^{n_{j} \times n_{j}} \forall j \in \mathbb{N}_{0}^{p-1}$, and the state-reference trajectory is denoted by $r_{j} \in \mathbb{R}^{n_{j}} \forall j \in \mathbb{N}_{0}^{p-1}$. Note that the above cost function was chosen because it suits the numerical example of Section 5 . A different cost function could be chosen without affecting the salient properties of the control law (see Remarks 7 and 8). The MPC problem is then given by Problem 6 .

Problem 6. Determine

$\bar{u}_{i}^{*}\left(x_{i}\right):=\arg \min _{\bar{u}_{i} \in \mathbb{R}^{\bar{m}_{\sigma(i)}}} J_{\sigma(i)}\left(x_{i}, \bar{u}_{i}\right)$

subject to prediction constraints (recall $x_{(i, 0)}:=x_{i}$ )

$E_{\sigma(i+k)} x_{(i, k)}+G_{\sigma(i+k)} u_{(i, k)} \leq b_{\sigma(i+k)} \forall k \in \mathbb{N}_{0}^{N-1}$

$x_{(i, 1)} \in \overline{\mathbb{C}}_{\sigma(i+1)}^{*}$

$x_{(i, k)} \in \mathbb{X}_{\sigma(i+k)} \quad \forall k \in \mathbb{N}_{2}^{N}$

and the following prediction dynamics for all $k \in \mathbb{N}_{0}^{N-1}$ :

$x_{(i, k+1)}=A_{\sigma(i+k)} x_{(i, k)}+B_{\sigma(i+k)} u_{(i, k)}+c_{\sigma(i+k)}$.
It holds that the periodic state-feedback MPC control law based on Problem 6 is a least-restrictive robust periodic control law according to Definition 5. This is because prediction constraint (4) explicitly constrains the closed-loop state trajectory to remain within the maximum robust periodic controlled invariant set, and furthermore because choosing the prediction state constraint sets $\mathbb{X}_{j}$ of (5) larger than $\mathbb{C}_{j}^{*}$ renders each element of the maximum robust periodic controlled invariant set feasible. This result is considered straightforward and thus, for brevity, it is stated without a formal proof.

\subsection{Robustly strongly feasible periodic MPC problem}

MPC Problem 6 is a robustly strongly feasible periodic MPC problem (Kerrigan, 2000). In words, an MPC problem is robustly strongly feasible if the closed-loop state trajectory $\left\{x_{i}\right\}_{i=j}^{\infty}$ from any feasible initial state $x_{j}$, due to any sequence $\left\{\bar{u}_{i}\right\}_{i=j}^{\infty}$ of feasible solutions to the MPC problem, and due to any sequence $\left\{w_{i} \in\right.$ $\left.\mathbb{W}_{\sigma(i)}\right\}_{i=j}^{\infty}$ of admissible disturbance realizations, remains within the sets of feasible states indefinitely. (See also Kerrigan, 2000.)

Remark 7. MPC problems that are robustly strongly feasible ensure that recursive feasibility is unrelated to optimality of the solution. Thus the MPC problem's objective cost function (3) can be tuned and changed in order to achieve a desirable response without risking potential constraint violation. (See also Remark 8.)

Robustly strongly feasible MPC problems are also useful if, e.g., an interior-point algorithms is terminated prior to natural termination to satisfy real-time constraints, or when an inaccurate solver is employed because, e.g., it is fast, memory-efficient, or requires little power.

\subsection{Discussion}

Robustness in MPC is usually enforced either by considering min-max open-loop predictions (Bemporad \& Morari, 1999) or by performing closed-loop predictions (Bemporad \& Morari, 1999; Goulart, Kerrigan, \& Maciejowski, 2006; Löfberg, 2003). The former generally results in very restrictive and conservative control laws, increasingly so for longer prediction horizons. The latter is generally less restrictive and conservative than the former, but results in much larger computational complexity. The approach proposed here employs the min-max open-loop paradigm over the first prediction step only and then performs nominal predictions (open-loop but not min-max) over the remaining prediction steps. Thus the (often crippling) conservativeness of min-max openloop predictions considered over the entire prediction horizon is avoided; in the proposed approach least-restrictiveness of the control law is maintained for every prediction horizon length. Note that the proposed approach results in no constraint violations, and furthermore, no relaxation or softening on the actual constraints is performed, only predicted. Note further that prediction dynamics (6) contain no mechanism to take into account, or exploit, the additive disturbances. Thus the number of decision variables of periodic MPC Problem 6 grows linearly with horizon length $N$. In contrast, the number of decision variables generally grows superlinearly with $N$ for closed-loop predictions, e.g., quadratically when employing affine disturbance-feedback (Goulart et al., 2006; Oldewurtel, Jones, \& Morari, 2008).

Any choice $\mathbb{X}_{j} \supseteq \mathbb{C}_{j}^{*} \forall j \in \mathbb{N}_{0}^{p-1}$, e.g., $\mathbb{X}_{j}=\mathbb{R}^{n_{j}} \forall j \in \mathbb{N}_{0}^{p-1}$, results in periodic MPC Problem 6 being robustly strongly feasible, and the resulting periodic control law least-restrictive. On the other hand a choice $\mathbb{X}_{j} \subset \mathbb{C}_{j}^{*}$ for some $j$ generally results in a control law that is not least-restrictive, even if the resulting MPC problem happens to be robustly strongly feasible. In the example of Section 5 we 
employ periodic MPC Problem 6 with $\mathbb{X}_{j}=\mathbb{C}_{j}^{*} \forall j \in \mathbb{N}_{0}^{p-1}$. Thus the predicted nominal state trajectory is constrained to a path that is admissible in closed-loop. By constraining the predicted state trajectory to lie within the maximum robust periodic controlled invariant set, at each prediction step the predicted state satisfies a necessary and sufficient condition for the existence of an admissible control input trajectory of infinite length. The finitehorizon MPC problem is thus expected to better approximate the infinite-horizon MPC problem than if $\mathbb{X}_{j} \supset \mathbb{C}_{j}^{*} \forall j \in \mathbb{N}_{0}^{p-1}$. However, for reducing the computation-times for solving periodic MPC Problem 6 it may be desirable to use low-complexity overapproximations of sets $\mathbb{C}_{j}^{*}$ as the prediction state constraint sets $\mathbb{X}_{j}$. The determination of low-complexity over-approximations is not discussed in this paper.

Computing maximum controlled invariant sets is challenging, and the difficulty in determining the maximum robust periodic controlled invariant set limits the applicability of the proposed approach. The required computations can generally only be performed exactly for state dimensions less than about 5 , somewhat higher in simple cases. Furthermore, as the dimension of the state increases, the solution of Problem 6 may become sensitive to the quality of the characterization of the invariant sets. Note that any non-maximum robust periodic controlled invariant set may be employed in the proposed MPC scheme, but may result in a control law that is not least-restrictive. The computation of low-complexity under-approximations of robust periodic controlled invariant sets is not discussed in this paper. Note that it is sometimes possible to circumnavigate the use of explicit characterizations of controlled invariant sets, by employing implicit equivalent conditions. This comes at the expense of a larger number of optimization variables in MPC Problem 6 (see Limon et al., 2012, details omitted for brevity).

\section{Building climate control example}

\subsection{Model of building and environment}

The objective is to minimize the energy consumption and/or cost of heating and cooling a building's rooms, while satisfying temperature constraints, imposed for occupants' comfort. The simplified plant model employed in Oldewurtel et al. (2008) (derived from Gwerder \& Tödtli, 2005) is depicted in Fig. 1. The system states are temperatures $t_{1}, t_{2}$ and $t_{3}$. The control inputs are $u_{\mathrm{h}}$ and $u_{\mathrm{c}}$. Environmental inputs $\delta_{1}, \delta_{2}$ and $\delta_{3}$ are not affected by the system. The heat capacities of nodes with temperature $t_{1}, t_{2}$ and $t_{3}$ are $C_{1}, C_{2}$ and $C_{3}$, respectively. The employed heat capacities $C_{k}$ and gains $K_{k}$ are for the simplified model of a room with $3600 \mathrm{~m}^{2}$ floor area (Gwerder \& Tödtli, 2005; Oldewurtel et al., 2008). The continuous-time dynamics are

$$
\begin{aligned}
\dot{t}_{1}= & \frac{1}{C_{1}}\left[\left(K_{1}+K_{2}\right)\left(t_{2}-t_{1}\right)+K_{5}\left(t_{3}-t_{1}\right)\right. \\
& \left.\quad+K_{3}\left(\delta_{1}-t_{1}\right)+u_{\mathrm{h}}+u_{\mathrm{c}}+\delta_{2}+\delta_{3}\right] \\
\dot{t}_{2}= & \frac{1}{C_{2}}\left[\left(K_{1}+K_{2}\right)\left(t_{1}-t_{2}\right)+\delta_{2}\right] \\
\dot{t}_{3}= & \frac{1}{C_{3}}\left[K_{5}\left(t_{1}-t_{3}\right)+K_{4}\left(\delta_{1}-t_{3}\right)\right] .
\end{aligned}
$$

This is expressed as the LTI system $\dot{x}=\mathscr{A} x+\mathscr{B} u+\mathcal{C} \omega$ with state $x:=\left[t_{1}, t_{2}, t_{3}\right]^{\top} \in \mathbb{R}^{3}$, input $u:=\left[u_{\mathrm{h}}, u_{\mathrm{c}}\right]^{\top} \in \mathbb{R}^{2}$, and environmental input $\omega:=\left[\delta_{1}, \delta_{2}, \delta_{3}\right]^{\top} \in \mathbb{R}^{3}$. Input constraints (7) are imposed at all times. State constraints (8) are time-dependent so that during business hours the controller provides a comfortable work environment. Outside of business hours rooms are allowed to cool or warm to reduce energy consumption. Note that a building $t_{1}$ : room air temperature to be controlled

$t_{2}$ : interior-wall surface temperature

$t_{3}$ : exterior-wall core temperature

$u_{\mathrm{h}}$ : heating power $(\geq 0)$

$u_{\mathrm{c}}$ : cooling power $(\leq 0)$

$\delta_{1}$ : outside air temperature

$\delta_{2}$ : solar radiation

$\delta_{3}$ : internal heat sources

$C_{1}=9.356 \cdot 10^{5} \mathrm{~kJ} /{ }^{\circ} \mathrm{C} \quad C_{2}=2.970 \cdot 10^{6} \mathrm{~kJ} /{ }^{\circ} \mathrm{C}$

$C_{3}=6.695 \cdot 10^{5} \mathrm{~kJ} /{ }^{\circ} \mathrm{C} \quad K_{1}=16.48 \quad \mathrm{~kW} /{ }^{\circ} \mathrm{C}$

$K_{2}=108.5 \quad \mathrm{~kW} /{ }^{\circ} \mathrm{C} \quad K_{3}=5 \quad \mathrm{~kW} /{ }^{\circ} \mathrm{C}$

$K_{4}=30.5 \quad \mathrm{~kW} /{ }^{\circ} \mathrm{C} \quad K_{5}=23.04 \quad \mathrm{~kW} /{ }^{\circ} \mathrm{C}$

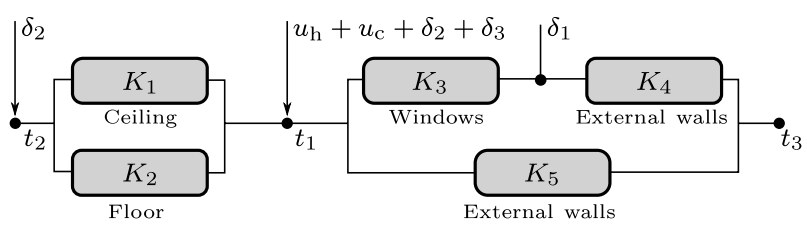

Fig. 1. Building climate control problem plant schematic.

controller's periodic schedule is usually fixed in advance. The temperature's comfort constraints are thus presumed fixed and not adjusted in real-time in response to feedback data on actual occupancy levels:

$0 \leq u_{\mathrm{h}} \leq 200, \quad-50 \leq u_{\mathrm{c}} \leq 0$

$\left.\begin{array}{l}21 \\ 19\end{array}\right\} \leq t_{1} \leq \begin{cases}26 & \text { from } 8 \text { am to } 6 \mathrm{pm} \\ 0 & \text { otherwise. }\end{cases}$

Environmental input $\omega$ consists of two parts such that $\omega=d+$ $w$, where $d$ is deterministic and periodically time-dependent with a period length of $24 \mathrm{~h}$, and $w$ is stochastic, bounded, and subject to periodically time-dependent bounds with a period length of $24 \mathrm{~h}$. The deterministic input $d$ is employed to model known fluctuations in environmental conditions, for example that the sun rises in the morning and sets in the evening, and that most internal heat sources (office workers, equipment, etc.) $\delta_{3}$ are active from $8 \mathrm{am}$ to $6 \mathrm{pm}$. The stochastic input $w$ is employed to yield robustness against unknown environmental conditions, for example because cloud cover affects the amount of solar radiation $\delta_{2}$ reaching the building, and because an office worker may either heat her lunch in the office microwave and continue working (increasing $\delta_{3}$ ) or switch off her PC and go to a restaurant (reducing $\delta_{3}$ ). The bounds on stochastic input $w$ are assumed known, but the stochastic properties of $w$ are unknown.

The LTI dynamics, constraints (7) and (8), deterministic environmental inputs $d$ and the bounds on stochastic environmental inputs $w$ are sampled with a zero-order hold and a sample-period of $10 \mathrm{~min}$, resulting in the periodic affine system (1) with a period length of $p=144$. This choice of sample-period length provides a useful compromise between temporal resolution of control input updates and computational complexity of the MPC problem when employing long prediction horizons. Note that this example uses time-invariant dimensions. The time-discretized deterministic environmental inputs $d$, bounds on stochastic environmental inputs $w$ and a realization of environmental inputs $\omega$ are plotted in Fig. 2 for one day. Values for $d$ and bounds on $w$ are based on real data (Gwerder \& Tödtli, 2005), but simplified. Stochastic inputs $w$ were randomly generated according to a uniform distribution. This stochastic disturbance model does not reflect reality and is employed here for simplicity. 


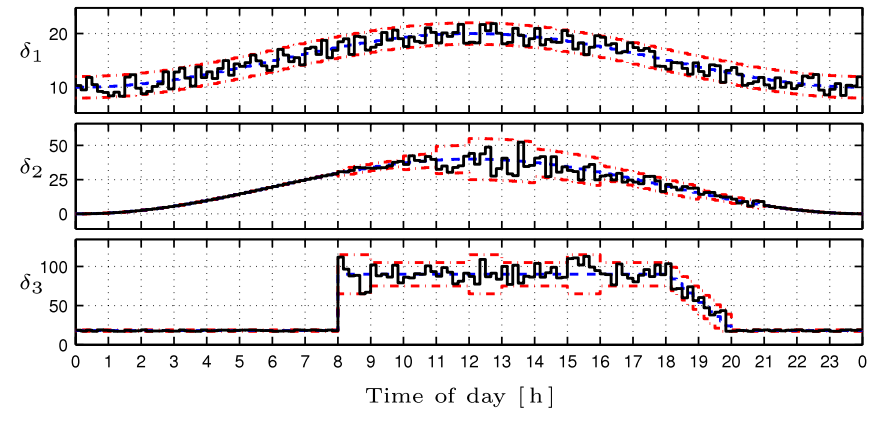

Fig. 2. Deterministic environmental inputs $d$ (dashed, blue), bounds on stochastic environmental inputs $w$ (dash-dotted, red), and realization of environmental inputs $\delta$ (solid, black). (For interpretation of the references to color in this figure caption, the reader is referred to the web version of this article.)

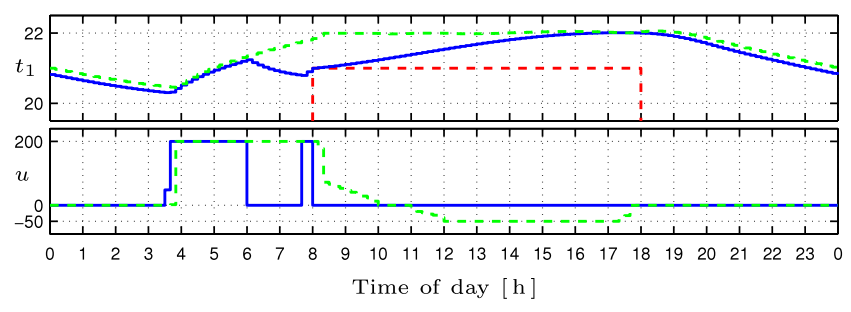

Fig. 3. Nominal periodic optimal solution: $q=0$ (solid, blue), $q=10^{5}$ (dashed, green). Constraint on $t_{1}$ (dashed, red). (For interpretation of the references to color in this figure caption, the reader is referred to the web version of this article.)

\subsection{MPC problem parameters}

The state's set-point is $r:=[22,0,0]^{\top}$. A room temperature of $t_{1}=22{ }^{\circ} \mathrm{C}$ is considered optimal (in this paper), whereas any value $21{ }^{\circ} \mathrm{C} \leq t_{1} \leq 26{ }^{\circ} \mathrm{C}$ is considered acceptable, for an office work environment. The cost matrices of (3) are chosen as follows with $q \in\left\{0,10^{5}\right\}$ :

$Q_{j}:= \begin{cases}\operatorname{diag}(q, 0,0) & \text { from } 8 \text { am to } 6 \mathrm{pm} \\ 0 & \text { otherwise }\end{cases}$

$R_{j}:= \begin{cases}{[2,-8]} & \text { from } 6 \text { am to } 10 \mathrm{pm} \\ {[1,-4]} & \text { otherwise. }\end{cases}$

When $q=0$ the control strategy is similar to zone-MPC (Ferramosca et al., 2010; Grosman et al., 2010; Maciejowski, 2002), and only input related costs are minimized. When $q=10^{5}$ the controller strives to maintain a $22{ }^{\circ} \mathrm{C}$ room temperature during business hours with little consideration for energy consumption. These two extreme values of $q$ are employed simply to demonstrate behavioral differences. The choice of $R_{j}$ models that energy prices during the day are twice those during the night; such peak/off-peak tariffs are common. Furthermore it reflects that cooling is more expensive (assumed four times) than heating, for the same power into/out of the room (Gwerder \& Tödtli, 2005). This is also reflected in constraints (7). Recall that $u_{\mathrm{c}}$ is negative, hence the minus sign in $R_{j}$.

The maximum robust periodic controlled invariant set was determined in $45 \mathrm{~s}$ on a $3.33 \mathrm{GHz}$ processor using the algorithm of Blanchini and Ukovich (1993), demonstrating the practicality of Assumption 4 for this example.

\subsection{Optimal nominal periodic control}

To obtain a baseline understanding of the form of the MPC solutions we first consider the solution to the nominal optimal periodic control problem, over the entire $24 \mathrm{~h}$ period, optimizing over both input trajectory and initial state, with an equality constraint between the initial and terminal states. Note that an equal initial and terminal state signifies that the one period solution is exactly repeatable, i.e., is equivalent to the multi-period solution. The solution is plotted in Fig. 3. When $q=0$ the controller preheats the room before 6 am to exploit cheaper energy prices and briefly applies heating before $8 \mathrm{am}$ to achieve the $21^{\circ} \mathrm{C}$ constraint at $8 \mathrm{am}$.

\subsection{Proposed vs. soft-constrained periodic MPC}

We first consider the zone-MPC approach with $q=0$. Plotted in Fig. 4(a) is the solution of the proposed MPC scheme with a unit length prediction horizon (10 $\mathrm{min}$ ). Despite the short prediction horizon the controller starts heating the room at 5:40 am, in order to satisfy the $21{ }^{\circ} \mathrm{C}$ constraint at $8 \mathrm{am}$. This is achieved because prediction constraints (4) and (5) provide the control law with all the information required to impose the minimum admissible room temperature at all times and indefinitely satisfy the constraints. Plotted in Fig. 4(b) is the solution of the proposed MPC scheme with a prediction horizon of 72 steps $(12 \mathrm{~h})$. This solution is now more similar to the periodic optimal solution plotted in Fig. 3.

All simulations' starting states are tuned such that in the nominal case the same state is achieved after $24 \mathrm{~h}$. As in Section 5.3, this implies that the nominal single-period solution is indicative of the nominal multi-period solution. In the disturbed case the single-period solution only repeats itself exactly if the disturbance sequence repeats itself exactly. While this is unlikely, due to the sluggish dynamics the disturbances do not strongly influence the closed-loop response, and thus the provided single-period plots do closely resemble the multi-period solution.

To demonstrate the benefits of the proposed MPC approach we next consider an MPC approach that does not enforce robust strong feasibility. It is not clear what the best method to compare with is, but we proceed as follows. We consider a nominal periodic MPC controller that, over its prediction horizon, takes into account only system constraints (2). As there is no provision for enforcing recursive feasibility the state constraints are considered soft, with

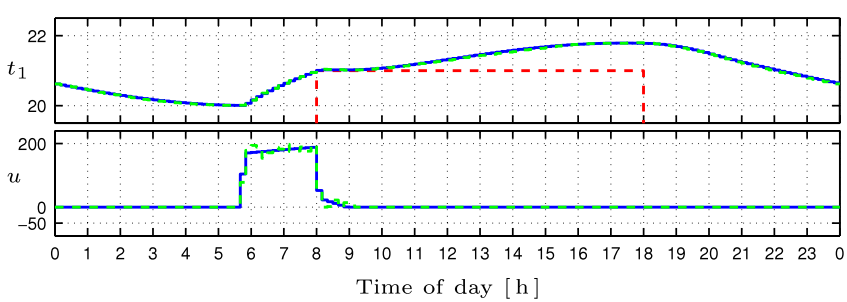

(a) Horizon length: $N=1(10 \mathrm{~min})$.

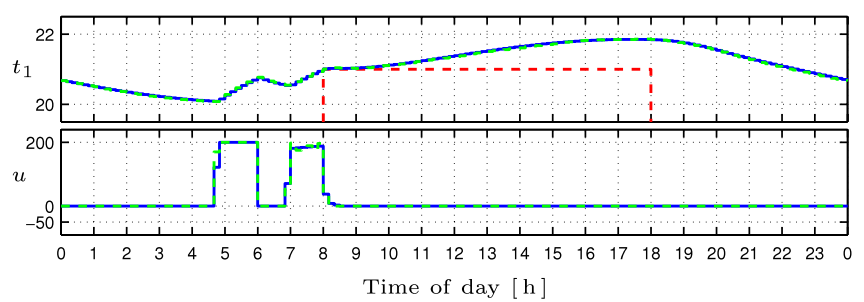

(b) Horizon length: $N=72(12 \mathrm{~h})$.

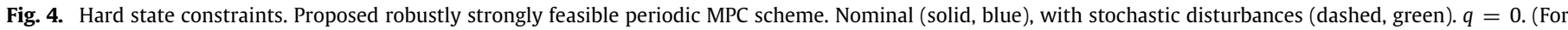
interpretation of the references to color in this figure caption, the reader is referred to the web version of this article.) 


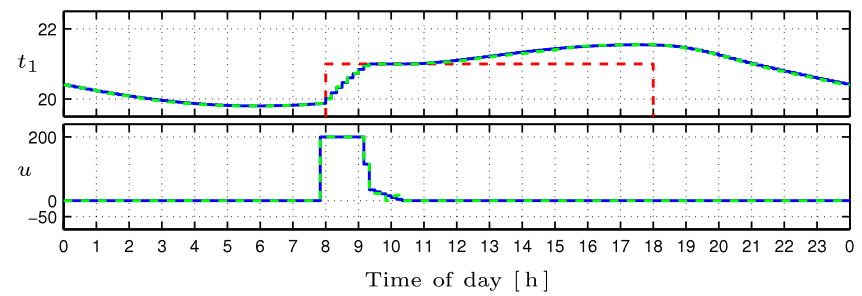

(a) Horizon length: $N=1(10 \mathrm{~min})$.

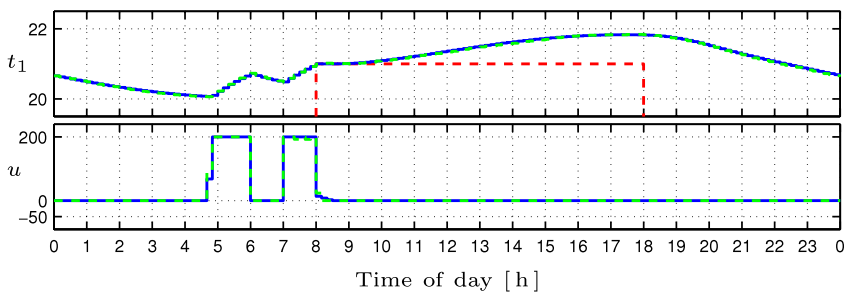

(b) Horizon length: $N=72(12 \mathrm{~h})$.

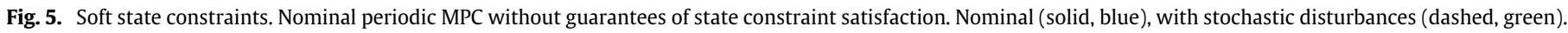
$q=0$. (For interpretation of the references to color in this figure caption, the reader is referred to the web version of this article.)

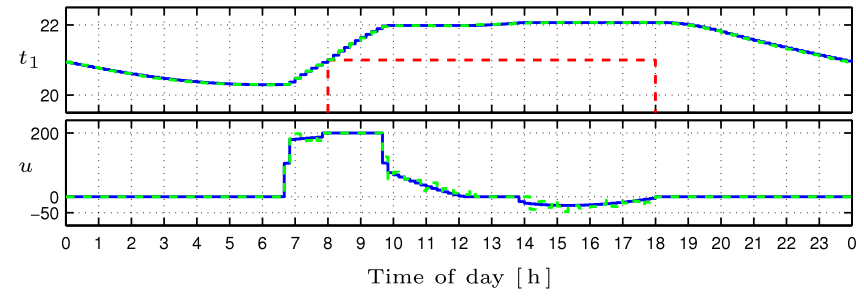

(a) Horizon length: $N=1(10 \mathrm{~min})$.

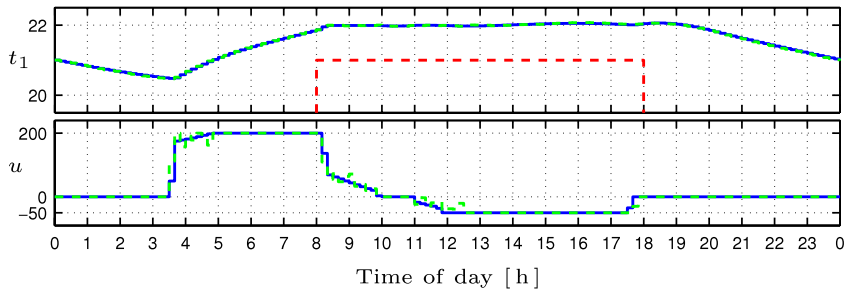

(b) Horizon length: $N=72(12 \mathrm{~h})$.

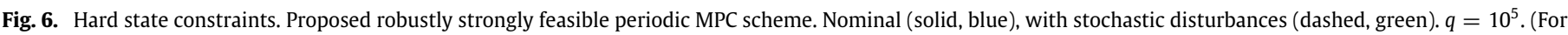
interpretation of the references to color in this figure caption, the reader is referred to the web version of this article.)

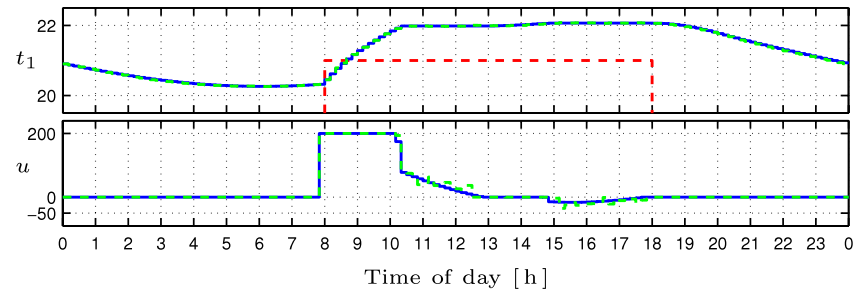

Fig. 7. Soft state constraints. Nominal periodic MPC without guarantees of state constraint satisfaction. Nominal (solid, blue), with stochastic disturbances (dashed, green). $q=10^{5}$. Plotted result: $N=1(10 \mathrm{~min})$. Result for $N=72(12 \mathrm{~h})$ is practically identical to Fig. 6(b), thus not plotted. (For interpretation of the references to color in this figure caption, the reader is referred to the web version of this article.)

a high penalty for violation, while input constraints remain hard. The result for a unit length prediction horizon (10 min) is plotted in Fig. 5(a). The controller only "sees" the 8 am $21{ }^{\circ} \mathrm{C}$ constraint at 7:50 am, due to the short horizon. A constraint violation occurs from 8 am until 9:20. The result for a 72 step $(12 \mathrm{~h})$ prediction horizon is plotted in Fig. 5(b) and looks very similar to the proposed MPC scheme's result of Fig. 4(b). In this case no constraint violations occur in the nominal case. All prediction horizon lengths $N \in \mathbb{N}_{1}^{72}$ were tested and it was found that the soft constrained approach leads to constraint violations for $N \leq 11$, whereas no constraint violations occur for $N \geq 12$, in the nominal case. The proposed robustly strongly feasible MPC approach requires no such trial-and-error analysis.

Results analogous to those plotted in Figs. 4 and 5, but for $q=10^{5}$, are plotted in Figs. 6 and 7. Again, despite a short prediction horizon the proposed robustly strongly feasible periodic MPC scheme enforces all constraints, whereas the soft-constrained alternative fails to do so. Note that in Fig. 7 the constraint violation lasts only until 8:40, shorter than in Fig. 5(a).

Remark 8. It is important to note that in the proposed MPC scheme the ability to enforce constraints, and the size of the feasible state space, is not related to, or dependent on, the prediction cost function of (3). In contrast, with the soft constrained MPC
Table 1

Daily energy consumption, energy cost and average room temperature for the eight MPC control laws contrasted in Figs. 4-7. Consumption and cost normalized by lowest value.

\begin{tabular}{rlllll}
\hline \multicolumn{1}{c}{$N$} & Hard & $q$ & $\begin{array}{l}\text { Normalized } \\
\text { consumption }\end{array}$ & $\begin{array}{l}\text { Normalized } \\
\text { cost }\end{array}$ & Average temp. $t_{1}$ \\
\hline 1 & $\checkmark$ & 0 & 1.396 & 1.320 & 21.012 \\
1 & $\times$ & 0 & $:=1$ & $:=1$ & 20.735 \\
72 & $\checkmark$ & 0 & 1.505 & 1.089 & 21.088 \\
72 & $\times$ & 0 & 1.473 & 1.072 & 21.066 \\
1 & $\checkmark$ & $10^{5}$ & 3.325 & 3.325 & 21.376 \\
1 & $\times$ & $10^{5}$ & 2.375 & 2.375 & 21.304 \\
72 & $\checkmark$ & $10^{5}$ & 7.301 & 6.549 & 21.576 \\
72 & $\times$ & $10^{5}$ & 7.301 & 6.549 & 21.576 \\
\hline
\end{tabular}

scheme employed for comparison here the cost function does affect the existence of, and the level of, constraint violations. Thus in the proposed scheme the control designer is free to tune cost function (3) to achieve desirable closed-loop behavior, without jeopardizing constraint satisfaction and feasibility.

\subsection{Discussion}

Performance values are tabulated in Table 1 . The proposed hard-constrained MPC strategy achieves its lowest energy consumption with a short prediction horizon, and its lowest energy cost with a long prediction horizon, both with $q=0$. More comfortable room temperatures, at much higher energy consumption and cost, are achieved with $q=10^{5}$. The soft-constrained MPC approach is able to achieve lower energy consumption and cost, at the expense of violating constraints.

\section{Conclusion}

Periodic affine systems provide a powerful modeling framework for a large variety of control problems, yet are straightforward to deal with. The ability to model not only periodic dynamics, but also periodic constraints, disturbances, disturbance bounds, 
external input variations, etc., is extremely powerful. The example of building climate control was used to convey the flexibility and convenience of the proposed approach. In many applications, e.g., the problem of room temperature control using a minimum amount of energy, the control performance is directly related to the ability to operate the plant close to the constraints, while rigorously enforcing constraint satisfaction. The consideration of leastrestrictive robust control laws is indispensable in these situations. By suitably tailoring the prediction state constraints for each prediction step, instead of employing usual terminal constraints, in the proposed periodic MPC problem formulation both the computational complexity of closed-loop prediction MPC, as well as the conservativeness of traditional min-max open-loop prediction MPC, were straightforwardly circumnavigated.

\section{References}

Bemporad, A., \& Morari, M. (1999). Robust model predictive control: A survey. Robustness in Identification and Control, 245, 207-226.

Bittanti, S., \& Colaneri, P. (2009). Periodic systems: Filtering and control. Springer.

Blanchini, F. (1999). Set invariance in control. Automatica, 35(11), 1747-1767.

Blanchini, F., \& Ukovich, W. (1993). Linear programming approach to the control of discrete-time periodic systems with uncertain inputs. Journal of Optimization Theory and Applications, 78(3), 523-539.

Boyd, S. P., \& Vandenberghe, L. (2004). Convex optimization. Cambridge University Press.

Ferramosca, A., Limon, D., González, A. H., Odloak, D., \& Camacho, E. F. (2010). MPC for tracking zone regions. Journal of Process Control, 20(4), 506-516.

Gondhalekar, R., \& Imura, J. (2010). Least-restrictive move-blocking model predictive control. Automatica, 46(7), 1234-1240.

Gondhalekar, R., \& Jones, C. N. (2011). MPC of constrained discrete-time linear periodic systems-A framework for asynchronous control: Strong feasibility, stability and optimality via periodic invariance. Automatica, 47(2), 326-333.

Goulart, P. J., Kerrigan, E. C., \& Maciejowski, J. M. (2006). Optimization over state feedback policies for robust control with constraints. Automatica, 42(4), 523-533.

Grosman, B., Dassau, E., Zisser, H. C., Jovanovič, L., \& Doyle III, F. J. (2010). Zone model predictive control: A strategy to minimize hyper- and hypoglycemic events. Journal of Diabetes Science and Technology, 4(4), 961-975.

Gwerder, M., \& Tödtli, J. (2005). Predictive control for integrated room automation. In 8th REHVA world congress for building technologies-CLIMA.

Kerrigan, E.C. (2000). Robust constraint satisfaction: Invariant sets and predictive control. Ph.D. Thesis, University of Cambridge, UK.

Limon, D., Alamo, T., Muñoz de la Peña, D., Zeilinger, M. N., Jones, C. N., \& Pereira, M. (2012). MPC for tracking periodic reference signals. In Proc. 4th IFAC NMPC conf., Netherlands.

Löfberg, J. (2003). Approximations of closed-loop minimax MPC. In 42nd IEEE CDC, USA.

Maciejowski, J. M. (2002). Predictive control with constraints. Prentice Hall.
Mayne, D. Q., Rawlings, J. B., Rao, C. V., \& Scokaert, P. (2000). Constrained model predictive control: Stability and optimality. Automatica, 36(6), 789-814.

Oldewurtel, F., Jones, C. N., \& Morari, M. (2008). A tractable approximation of chance constrained stochastic MPC based on affine disturbance feedback. In 47th IEEE $C D C$, Mexico.

Varga, A. (2004). Computation of minimal periodic realizations of transfer-function matrices. IEEE Transactions on Automatic Control, 49(1), 146-149.

Varga, A. (2007). An overview of recent developments in computational methods for periodic systems. In IFAC workshop on periodic control systems, Russia.

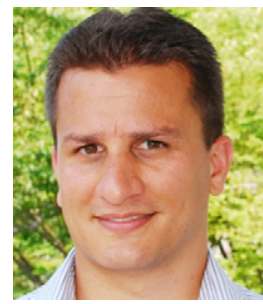

Ravi Gondhalekar is a Project Scientist at the University of California Santa Barbara, USA. His research interests include model predictive control, constrained control and optimization, and the application of constrained model predictive control techniques to an artificial pancreas that performs automated delivery of insulin to people with type 1 diabetes. Ravi received a Ph.D. degree in informatics in 2008 from the Tokyo Institute of Technology, Japan, and M.Eng. and B.A. degrees in engineering in 2002 from the University of Cambridge, UK. From 2008 to 2012 Rav was a tenure-track Assistant Professor at Osaka University, Japan. Prior to that he held short-term positions at the Massachusetts Institute of Technology, USA, the University of Cambridge, UK, Princeton University, USA, Pi Technology, UK, the Rutherford Appleton Laboratory, UK, and the United Kingdom Atomic Energy Authority, UK.

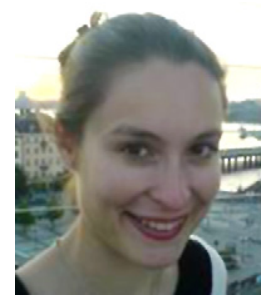

Frauke Oldewurtel is a Postdoctoral Researcher at the Power Systems Laboratory of the Swiss Federal Institute of Technology Zurich (ETHZ), Switzerland. She obtained a Ph.D. in 2011 at the Automatic Control Laboratory at ETH Zurich for her work on stochastic model predictive control for energy efficient building climate control. She received a diploma in Electrical Engineering and Business Administration from the Technical University of Braunschweig, Germany, in 2006, and a Master of Science from Georgia Institute of Technology, USA, in 2004. Her current research focuses on stochastic control and optimization with application to electricity grids and buildings.

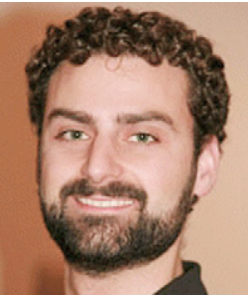

Colin N. Jones is an Assistant Professor in the Automatic Control Laboratory at the École Polytechnique Fédérale de Lausanne (EPFL) in Switzerland. He was a Senior Researcher at the Automatic Control Lab at ETH Zurich until 2010 and obtained a Ph.D. in 2005 from the University of Cambridge for his work on polyhedral computational methods for constrained control. Prior to that, he was at the University of British Columbia in Canada, where he took a bachelor and master in Electrical Engineering and Mathematics. His current research interests are in the areas of high-speed predictive control and optimization, as well as green energy generation, distribution and management. 